\title{
The role of echocardiography in prognosis for dysfunction and abandonment of radiocephalic arteriovenous fistula in elderly Chinese patients on hemodialysis
}

\author{
Yuqin Xiong $^{1}$ | Yang Yu ${ }^{1,2}$ | Chunle Zhang ${ }^{1}$ | Emily Morris ${ }^{3}$ | Liya Wang ${ }^{1}$ | \\ Yuchen Deng $^{1}$ | Yi Li ${ }^{3}$ | Ping Fü
}

${ }^{1}$ Kidney Research Laboratory, Division of Nephrology, West China Hospital of Sichuan University, Chengdu, China

${ }^{2}$ National Clinical Research Center for Geriatrics, West China Hospital of Sichuan University, Chengdu, China

${ }^{3}$ Department of Biostatistics, University of Michigan, Ann Arbor, USA

\section{Correspondence}

Ping Fu, Kidney Research Laboratory, Division of Nephrology, West China Hospital of Sichuan University, No.37, Guoxue lane, Wuhou District, Chengdu, Sichuan Province, 610041, China.

Email: fupinghx@scu.edu.cn

Funding information

This work was financially supported by the 1.3.5 Project for Disciplines of Excellence, West China Hospital, Sichuan University and the Research Program of Health Commission of SiChuan Province (18PJ362).

\begin{abstract}
The objective of this study was to examine the impact of cardiac structure and function at baseline on the outcomes associated with arteriovenous fistula (AVF) in patients on hemodialysis (HD). Patients who initiated $\mathrm{HD}$ aged $\geq 70$ years and received a mature AVF creation were included retrospectively. Echocardiographic parameters measured within 1 week before AVF creation were acquired. The observational period for each patient was from the point of AVF creation to the last time of follow-up unless AVF abandonment or death occurred. Kaplan-Meier and Cox proportional hazard regression analyses were conducted. A total of 82 elderly Chinese HD patients with mature radiocephalic AVF (RCAVF) and EF $\geq 50 \%$ were analyzed. During the median study period of 26.8 (1240) months, 42 (51.2\%) experienced RCAVF dysfunction and 34 (41.5\%) progressed to abandonment. Primary and cumulative patencies at $6,12,24$, and 36 months were $81 \%$, $73 \%, 48 \%, 38 \%$, and $84 \%, 81 \%, 68 \%, 55 \%$, respectively. Left ventricle end-diastolic volume (LVEDV) $\leq 103.5 \mathrm{~mL}(\mathrm{HR}=2.5, P=.019)$ and the right side of RCAVF (HR = 3.59, $P=.003)$ significantly predicted RCAVF dysfunction. The main pulmonary artery internal diameter (MPAID) $\leq 21.5 \mathrm{~mm}(\mathrm{HR}=4.3, P=.001)$ as well as the right side $(\mathrm{HR}=2.95$, $P=.047)$ were the independent predictors for RCAVF abandonment. In conclusion, LVEDV, MPAID assessed by echocardiography and the right side of RCAVF, showed significant predictive implications for the outcomes of RCAVF. Disparities among nationalities in the areas of utilization and patency of AVFs necessitate additional studies.
\end{abstract}

\section{1 | INTRODUCTION}

Aging populations have led to an increasing number of end stage renal disease (ESRD) patients on maintenance hemodialysis (HD). ${ }^{1}$ Current studies suggest arteriovenous fistula (AVF) as the preferred vascular access (VA) type for elderly HD patients due to longer patency and lower incidence of VA-related infection. ${ }^{2,3}$

It has been widely acknowledged that ventricular remodeling and dysfunction, assessed by echocardiography, develop following AVF

Yuqin Xiong and Yang Yu contributed equally to this work. creation and dialysis initiation. Furthermore, the risk of pulmonary hypertension (PAH) due to high-flow rate of AVF was increased despite improved left ventricular (LV) pressure and volume load through dialysis. ${ }^{4-6}$ This, raised the issue that what kind of cardiac structure and function patients had at baseline, could tolerate the adverse effects derived from AVF and provide the AVF with adequate blood flow for HD treatment. To date, few studies have identified the association between preoperative echocardiography and the outcomes of AVF.

Although preoperative retention of temporary catheter was thought to be correlated with lower patency of $\mathrm{AVF}^{3,7}$ it remains unclear whether the previous modality and location of VA also 
contribute to the outcomes of AVF. In addition, different regions have varied requirements on dialysis flow, which could result in a potential discrepancy in functional life of AVFs. ${ }^{8,9}$ Related data for elderly Chinese ESRD patients have been scarce.

Based on the above, we designed a retrospective study and aimed to: (a) explore the prognostic value of echocardiographic indexes and prior VA history for the outcomes of AVF; and (b) report the utilization and patency of AVFs for elderly HD patients in China.

\section{2 | MATERIALS AND METHODS}

\section{1 | Study population}

We retrospectively reviewed ESRD patients aged $\geq 70$ years who were admitted to our hospital and obtained a mature AVF creation.

Patients were excluded when: (a) they initiated HD before the age of 70; (b) their ejection fraction (EF) $<50 \%$ or echocardiographic parameters were incomplete before AVF creation; (c) they underwent HD combined with, or switched to, peritoneal dialysis; (d) they died from any cause during the hospitalization of AVF creation; (e) the dialysis remained dependent on other VAs for an unmatured AVF; (f) they had not initiated HD yet or withdrew from HD due to stable renal function based on maintenance medication until last follow-up, even if the AVFs achieved maturation; (g) they were lost to contact, or they refused to be investigated.

\subsection{Clinical data collection and definitions}

Clinical data were collected through electronic medical records, including demographic characteristics, etiology of ESRD, coexisting conditions, echocardiographic parameters within 1 week prior to AVF creation, preoperative HD status, and the location of AVF.

For each patient, the patency, dysfunction, intervention, and abandonment of the AVF were acquired by reviewing the patient's readmission records and initiating phone calls to patients' homes. The last follow-up was defined as the point of last contact at a dialysis center, outpatient clinic, inpatient ward or through phone call. The study period for each patient was from the point of AVF creation to the last time of follow-up unless AVF abandonment or death occurred. Maturation, ${ }^{8}$ primary and cumulative patency, AVF abandonment ${ }^{10}$ were defined in accordance with published standards. In addition, serum biochemical indices were excluded from the analysis, because the concentration of serum biomarkers on entry may not represent the actual level as some patients underwent HD or blood transfusion treatment before admission to our clinic.

\section{3 | Statistical analysis}

Patients were assigned to groups according to the dysfunction or abandonment of AVF during the study period. The continuous variables are presented as mean (standard deviation), or median (interquartile range) when the values did not show a normal distribution. Categorical variables are presented as percentages. The Kruskal-Wallis $\mathrm{H}$ test was used for continuous variables, while the chi-square test was applied to assess the categorical data.

Receiver operating characteristic (ROC) curves were generated to determine the optimal cut-off value. Kaplan-Meier curves were utilized to demonstrate differences in survival. Log rank tests were performed to examine the significance of the difference between the curves. Variables with $P<.05$ in the univariate analysis and those considered clinically important were entered into Cox proportional hazards regression models to explore predictors for outcomes of AVF. A value of $P<.05$ was regarded as an acceptable threshold for significance. Data were analyzed using SPSS version 22.0.

\section{3 | RESULTS}

\section{1 | Inclusion of subjects and follow-up procedure}

One hundred and eighty HD patients aged $\geq 70$ years with a total of 220 AVF surgeries at our hospital from January 2007 to October 2018 were screened through electronic medical records by two investigators. Among these surgeries, 143 AVFs achieved maturation, 66 AVFs failed to mature, and the remaining 11 failed immediately (no murmur or tremor was detected within 72 hours after AVF creation).

The final follow-up dates were observed between January 2019 and March 2019. After excluding 98 patients, 82 eligible participants with a median follow-up period of 26.8 (12-40) months were included in the final analysis. All of them received radiocephalic AVFs (RCAVFs).

\subsection{Characteristics at baseline and outcomes of RCAVFs}

Among the 82 elderly HD patients, 42 (51.2\%) experienced RCAVF dysfunction and 34 (41.5\%) progressed to abandonment. The baseline clinical, echocardiographic, and previous VA and RCAVF characteristics of the subjects were summarized in Tables 1 and 2 . There was no history of peripheral vascular disease (PVD) or arteriovenous graft in this cohort.

In terms of patency for the mature RCAVFs, primary and cumulative patencies at $6,12,24$, and 36 months were $81 \%, 73 \%$, $48 \%, 38 \%$, and $84 \%, 81 \%, 68 \%, 55 \%$, respectively. As for the cause of RCAVF dysfunction, 38 (90.5\%) patients had AVF stenosis, 4 (9.5\%) suffered from venous hypertension syndrome or central venous stenosis. In regard to intervention, 13 cases of balloon angioplasty, 1 case of balloon angioplasty + stent deployment, and 8 cases of surgical revision were performed for 15 patients. 
TAB LE 1 Characteristics in groups according to RCAVF abandonment

\begin{tabular}{|c|c|c|c|c|}
\hline Characteristic & Total & Abandoned & No abandoned & $P^{a}$ \\
\hline No. of patients & 82 & 34 & 48 & - \\
\hline Age (year) & $73(71-75)$ & $74(71-76)$ & $73(71-75)$ & 0.29 \\
\hline Etiology of ESRD no. (\%) & & & & 0.61 \\
\hline Diabetic nephropathy & 27 (32.9) & $13(38.2)$ & $14(29.2)$ & - \\
\hline Others & $18(22)$ & $6(17.7)$ & $12(25)$ & - \\
\hline \multicolumn{5}{|l|}{ Previous medical history no. (\%) } \\
\hline Diabetes mellitus & $40(48.8)$ & $14(41.2)$ & $26(54.2)$ & 0.25 \\
\hline Congestive heart failure & $5(6.1)$ & $2(5.9)$ & $3(6.3)$ & 0.95 \\
\hline Coronary heart disease & $3(3.7)$ & $2(5.9)$ & $1(2.1)$ & 0.37 \\
\hline \multicolumn{5}{|l|}{ Preoperative assessment } \\
\hline Diastolic blood pressure $(\mathrm{mm} \mathrm{Hg})$ & $75.9(11.2)$ & $74.7(7.7)$ & $76.8(13.2)$ & 0.01 \\
\hline Ejection fraction (\%) & $66.1(5.5)$ & $66.7(5.9)$ & $65.7(5.3)$ & 0.37 \\
\hline LAID (mm) & $38.7(5.2)$ & $38.5(5.9)$ & $38.8(4.6)$ & 0.18 \\
\hline RAID (mm) & $36(33-39)$ & $35(33-38)$ & 37 (34-39) & 0.28 \\
\hline $\operatorname{LVID}(\mathrm{mm})$ & $48(46-52)$ & $47(45-49)$ & $49(46-54)$ & 0.012 \\
\hline $\mathrm{RVID}(\mathrm{mm})$ & $21(20-23)$ & $20(20-22)$ & $22(20-23)$ & 0.05 \\
\hline AAOID (mm) & $34.3(3.4)$ & $34(3.7)$ & $34.4(3.2)$ & 0.47 \\
\hline MPAID (mm) & $22(21-24)$ & $21(20-23)$ & $23(21-26)$ & 0.001 \\
\hline $\operatorname{LVESV}(\mathrm{mL})$ & $34.5(29-44)$ & $30(27-40)$ & $36.5(32-48)$ & 0.004 \\
\hline Immediate AVFs failure no. (\%) & $4(4.9)$ & $3(8.8)$ & $1(2.1)$ & 0.16 \\
\hline AVF maturation failure no. (\%) & $6(7.3)$ & $4(11.8)$ & $2(4.2)$ & 0.19 \\
\hline Duration of FV-ntCVCs (mo) & $0(0-2)$ & $0.75(0-2.5)$ & $0(0-0.7)$ & 0.036 \\
\hline Duration of IJV-ntCVCs (mo) & $0(0-2)$ & $0(0-0)$ & $0(0-2.2)$ & 0.025 \\
\hline Length of previous dialysis (mo) & $0.2(0-0.7)$ & $0.1(0-0.5)$ & $0.2(0-0.7)$ & 0.45 \\
\hline \multicolumn{5}{|l|}{ Outcomes of current RCAVF } \\
\hline Right RCAVF no. (\%) & $19(23.2)$ & $11(32.4)$ & $8(16.7)$ & 0.1 \\
\hline Maturation period (mo) & $2.5(2-3)$ & $2.5(2-3)$ & $2.5(2-3)$ & 0.79 \\
\hline Primary patency (mo) & $23.8(11-31)$ & $14.5(8-27)$ & $26.5(14.5-38.5)$ & 0.002 \\
\hline No. of interventions ${ }^{b}$ & $0(0-4)$ & $0(0-4)$ & $0(0-3)$ & 0.27 \\
\hline Length of follow-up (mo) & $26.8(12-40)$ & $19.5(9-39)$ & $27.5(21.8-41.8)$ & 0.045 \\
\hline
\end{tabular}

Abbreviations: AAOID, ascending aorta internal diameter; FV, femoral vein; IJV, internal jugular vein; LAID, left atrium internal diameter; left ventricle stroke volume; LVEDV, left ventricle end-diastolic volume; LVESV, left ventricle end-systolic volume; LVID, left ventricle internal diameter; LVSV;MPAID, main pulmonary artery internal diameter; ntCVC, nontunneled central venous catheter; PVA, permanent vascular access; RAID, right atrium internal diameter; RCAVF, radiocephalic arteriovenous fistula; RVID, right ventricle internal diameter; tcCVC, tunneled cuffed central venous catheter.

${ }^{\text {a }} P$ value for comparison among two groups excluding the total group.

${ }^{b}$ Variables are presented as median (full range) since their median (interquartile range) are both 0 (0-0). 


\section{3 | Predictors for outcomes of RCAVF in Cox hazard regression analysis}

According to the results of univariate (Table 1, 2) and ROC (data not listed) analyses, LV internal diameter (LVID), main pulmonary artery internal diameter (MPAID), LV end-systolic volume (LVESV), LV end-diastolic volume (LVEDV), and LV stroke volume (LVSV) showed significant differences between groups. Because of the correlation among the three values (LVSV = LVEDV-LVESV), only LVID, MPAID, and LVEDV were selected to be included in the regression models.

As presented in Table 3, LVEDV $\leq 103.5 \mathrm{~mm} \mathrm{Hg}$ and the right side of operations were independent predictors for RCAVF dysfunction. Moreover, the survival curve of RCAVFs with LVEDV $>103.5 \mathrm{~mm}$ (Figure 1 ) indicated longer survival times compared to those with LVEDV $\leq 103.5(P=.005)$

For RCAVF abandonment, MPAID (whether presented as a continuous or categorical variable according to the optimal cut-off value of $\leq 21.5 \mathrm{~mm}$ ), and the right side acted as significant predictors in the two Cox regression analyses (Table 4 model $a$ and b). Furthermore, the survival of RCAVFs in the group with MPAID >21.5 mm (Figure 2) showed better outcomes than the converse group.

\section{4 | DISCUSSION}

\section{1 | Echocardiography and surgical site in predicting outcomes of RCAVF}

In the present study on 82 Chinese ESRD patients who initiated HD aged $\geq 70$ years, the factors involving previous VAs, reflected not only the dialysis vintage but also a potential proneness to vascular intimal hyperplasia $(\mathrm{IH})$ and thrombosis. These factors were adjusted when we explored the predictors for RCAVF dysfunction and abandonment.

In the cohort with normal cardiac systolic function ( $E F \geq 50 \%$ ), LVEDV and the right side of RCAVF were independent predictors

\begin{tabular}{|c|c|c|c|c|}
\hline Characteristic $^{a}$ & Total & Unusable & Usable & $P^{b}$ \\
\hline No. of patients & 82 & 42 & 40 & - \\
\hline Age (year) & $73(71-75)$ & $74(71-76)$ & $72.5(71-75)$ & .44 \\
\hline Male no. (\%) & $49(59.8)$ & $22(52.4)$ & $27(67.5)$ & .16 \\
\hline \multicolumn{5}{|l|}{ Preoperative assessment } \\
\hline $\begin{array}{l}\text { Systolic blood } \\
\text { pressure }(\mathrm{mm} \mathrm{Hg})\end{array}$ & $146(130-155)$ & $146.5(124-155)$ & $146(134-157)$ & .45 \\
\hline $\begin{array}{l}\text { Diastolic blood } \\
\text { pressure }(\mathrm{mm} \mathrm{Hg})\end{array}$ & $75.9(11.2)$ & $74.8(9.4)$ & $77.1(12.9)$ & .024 \\
\hline $\operatorname{LVID}(\mathrm{mm})$ & $48(46-52)$ & $47(45-49)$ & $50(47-54)$ & .006 \\
\hline LVESV (mL) & $34.5(29-44)$ & $30(28-41)$ & $36(32-51)$ & .01 \\
\hline LVEDV $(\mathrm{mL})$ & $107(94-127)$ & $100(91-112)$ & $112(99-141)$ & .009 \\
\hline $\operatorname{LVSV}(\mathrm{mL})$ & $70(62-86)$ & $67(61-81)$ & $76(67-94)$ & .011 \\
\hline \multicolumn{5}{|c|}{ Previous vascular access history } \\
\hline No. of $P V A^{c}$ & $0(0-3)$ & $0(0-2)$ & $0(0-3)$ & .38 \\
\hline $\begin{array}{l}\text { Functional length of } \\
\operatorname{tcCVCs} s^{c}(\mathrm{mo})\end{array}$ & $0(0-72)$ & $0(0-17)$ & $0(0-72)$ & .17 \\
\hline $\begin{array}{l}\text { Functional length of } \\
\mathrm{AVFs}^{\mathrm{c}}(\mathrm{mo})\end{array}$ & $0(0-84)$ & $0(0-84)$ & $0(0-51)$ & .76 \\
\hline $\begin{array}{l}\text { Duration of FV- } \\
\text { ntCVCs (mo) }\end{array}$ & $0(0-2)$ & $0.28(0-2.3)$ & $0(0-0.6)$ & .06 \\
\hline $\begin{array}{l}\text { Duration of IJV- } \\
\text { ntCVCs (mo) }\end{array}$ & $0(0-2)$ & $0(0-1)$ & $0(0-2.2)$ & .16 \\
\hline Right RCAVF (\%) & $19(23.2)$ & $14(33.3)$ & $5(12.5)$ & .025 \\
\hline $\begin{array}{l}\text { Length of follow-up } \\
\text { (mo) }\end{array}$ & $23.8(11-31)$ & $16.5(8-28)$ & $26(14.5-39.5)$ & .007 \\
\hline
\end{tabular}

TABLE 2 Characteristics in groups according to RCAVF dysfunction

Abbreviations: FV, femoral vein; IJV, internal jugular vein; left ventricle stroke volume; LVEDV, left ventricle end-diastolic volume; LVESV, left ventricle end-systolic volume; LVID, left ventricle internal diameter; LVSV, left ventricle stroke volume;ntCVC, nontunneled central venous catheter; PVA, permanent vascular access; RCAVF, radiocephalic arteriovenous fistula; tcCVC, tunneled cuffed central venous catheter.

${ }^{a}$ Variables with $P<.05$ in the comparison or those considered clinically important are listed.

${ }^{\mathrm{b}} P$ value for comparison among two groups excluding the total group.

${ }^{\complement}$ Variables are presented as median (full range) since their median (interquartile range) are both $0(0-0)$. 
TABLE 3 Cox proportional hazard regression models for RCAVF dysfunction

\begin{tabular}{|llll|}
$\begin{array}{l}\text { Variable (per } 1 \text { unit increased) or } \\
\text { (yes vs no) }\end{array}$ & HR & $95 \% \mathrm{Cl}$ & P \\
\hline Male & 1.2 & $0.58-2.47$ & .63 \\
\hline Age (year) & 1.06 & $0.96-1.17$ & .23 \\
\hline Diabetes mellitus & 0.81 & $0.41-1.58$ & .53 \\
\hline DBP (mm Hg) & 0.97 & $0.94-1$ & .08 \\
\hline LVID (mm) $\leq 48.5$ & 1.8 & $0.76-4.24$ & .18 \\
\hline LVEDV (mL) $\leq 103.5$ & 2.5 & $1.17-5.37$ & .019 \\
\hline Previous No. of PVA & 0.63 & $0.22-1.76$ & .38 \\
\hline $\begin{array}{l}\text { Previous functional length of AVFs } \\
\text { (mo) }\end{array}$ & 1.01 & $0.98-1.05$ & .5 \\
\hline Previous functional length of & 1 & $0.89-1.12$ & .99 \\
\hline tcCVCs (mo) & & & \\
\hline Previous duration of FV-ntCVC (mo) & 1.14 & $0.92-1.41$ & .25 \\
\hline Previous duration of IVJ-ntCVC (mo) & 0.92 & $0.75-1.13$ & .42 \\
\hline Right RCAVF & 3.59 & $1.54-8.39$ & .003 \\
\hline
\end{tabular}

Abbreviations: DBP, diastolic blood pressure; FV, femoral vein; $\mathrm{HR}$, Hazard rate; $\mathrm{Cl}$, confidence interval; IJV, internal jugular vein; LVEDV, left ventricle end-diastolic volume; LVID, left ventricle internal diameter; ntCVC, nontunneled central venous catheter; PVA, permanent vascular access; RCAVF, radiocephalic arteriovenous fistula; tcCVC, tunneled cuffed central venous catheter.

Overall (score) $\chi^{2}=26.65, P=.009$.

for dysfunction. Patients with LVEDV $\leq 103.5 \mathrm{~mL}$ possessed 2.5 times increased risk compared to those with LVEDV $>103.5 \mathrm{~mL}$ (Table 3). This might indicate an insufficient cardiac diastolic function for the gradually increased blood volume flowing back to the heart after RCAVF creation, which in turn intensified the pressure of AVF outflow tract and restricted its flow. The sustaining pressure could induce $\mathrm{IH}$ or stenosis of the whole HD access circuit, thereby promoting the development of AVF dysfunction.

Our clinic typically chose nondominant upper extremity (mostly left arm in this cohort) as the surgical site in keeping with guidelines, ${ }^{8}$ the exceptional employments of right arm in 19 patients implied their poor overall vascular conditions. Besides, previous placements of right internal jugular vein catheter were prone to causing proximal traumatic stenosis of the ipsilateral AVF circuit, which might impair the function of right RCAVF. Therefore, it makes sense that the risk for RCAVF dysfunction and abandonment on the right side showed over three times greater risk than the left (Tables 3 and 4).

In addition to surgical site, MPAID acted as a strong predictor for RCAVF abandonment in the population without $\mathrm{PAH}$ at baseline. MPAID $\leq 21.5 \mathrm{~mm}$ before AVF creation had over fourfold the risk for AVF abandonment as those whose MPAID was larger, with the risk decreasing by $32 \%$ per each $1 \mathrm{~mm}$ elevation (Table 4). As a structural indicator of the heart, smaller MPAID might suggested an irreversible low tolerance to the increasing blood flow that returned to the heart. Since the occlusion of AVF could reverse the deterioration

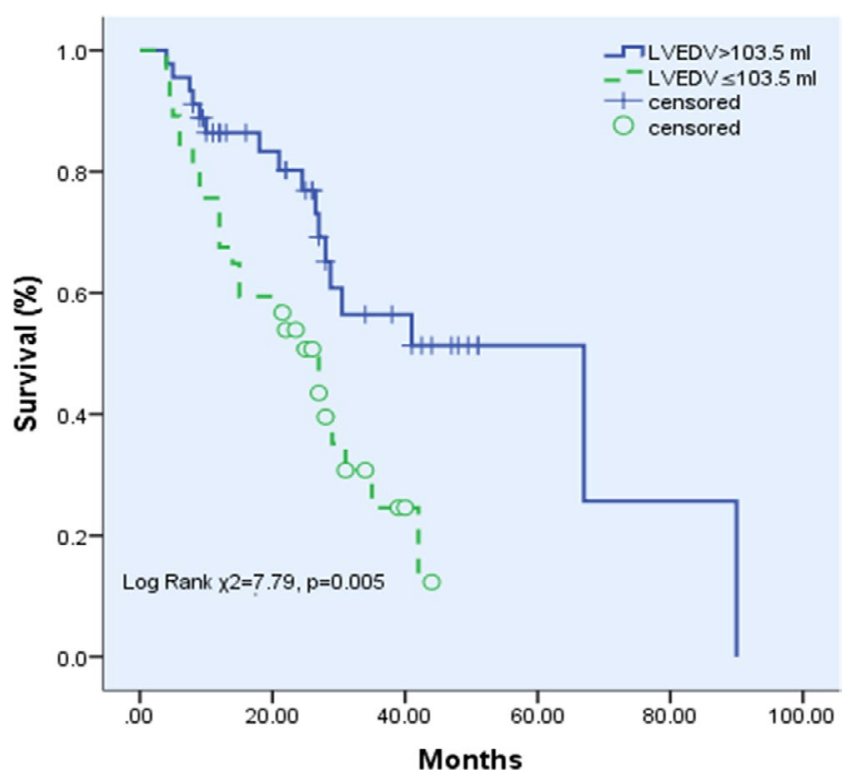

FIGURE 1 Curves respectively illustrating the cumulative functional survival (equal to primary patency) of RCAVF over time in groups according to the value of LVEDV. LVEDV, left ventricle end-diastolic volume [Color figure can be viewed at wileyonlinelibrary.com]

of cardiac function, ${ }^{11}$ and most of abandoned AVFs in this cohort converted to other types of VA in further observation, ${ }^{12}$ prognostic analysis of echocardiographic parameters or AVF on cardiovascular outcome was not conducted in this study.

\section{2 | Patencies and outcomes of RCAVF in Chinese ESRD patients aged $\geq 70$ years}

A maturation rate of $68.4 \%$ was calculated in the 209 AVFs. RCAVF was the more common choice for the aged in our clinic compared to brachiocephalic AVF (BCAVF). This was mainly due to concerns about the greater possibility that high-flow AVF formation increased the risk for high-output cardiac failure. ${ }^{11,13-15}$ Even though BCAVF was reported with higher patency, ${ }^{16}$ several centers published excellent results for RCAVFs in the elderly. ${ }^{17}$

Patients in the study demonstrated superior primary patencies compared to their counterparts aged $\geq 70-75$ years ( $54 \%$ at 12 months for British, $73.1 \%$ at 6 months and $57.1 \%$ at 12 months for Koreans; $39.7 \%$ at 12 months and $31.2 \%$ at 24 months for Americans), and also better cumulative patencies (66\% at 12 months for British; $56 \%$ at 12 months and $51 \%$ at 24 months for Americans). ${ }^{2,18,19}$ Observed values were much closer to a general Chinese ESRD population, ${ }^{7}$ confirming the regional differences. Our Chinese cohort had longer maturation time and greater patency compared with the United States or Europe, ${ }^{8,9}$ which was consistent with previous studies showing an inverse correlation between maturation time and AVF failure. ${ }^{20,21}$ Also, the lower routine blood flow $(200-300 \mathrm{~mL} / \mathrm{min})$ during HD treatment in China, might preserve the function and lifetime of AVF. Another reason that might contribute to these differences was that 


\begin{tabular}{|c|c|c|c|c|c|c|}
\hline $\begin{array}{l}\text { Variable (per } 1 \text { unit increased) } \\
\text { or (yes vs no) }\end{array}$ & HR (a) & $95 \% \mathrm{Cl}$ & $P$ & $\mathrm{HR}(\mathrm{b})$ & $95 \% \mathrm{Cl}$ & $P$ \\
\hline Male & 0.48 & $0.19-1.21$ & .12 & 0.61 & $0.26-1.42$ & .25 \\
\hline Age (year) & 1.04 & $0.94-1.16$ & .48 & 1.06 & $0.95-1.17$ & .29 \\
\hline Diabetes mellitus & 0.99 & $0.44-2.21$ & .98 & 1.2 & $0.55-2.63$ & .65 \\
\hline $\mathrm{DBP}(\mathrm{mm} \mathrm{Hg})$ & 0.98 & $0.93-1.03$ & .37 & 0.98 & $0.94-1.03$ & .48 \\
\hline LVID (mm) & 1.05 & $0.89-1.23$ & .57 & 1.07 & $0.92-1.26$ & .39 \\
\hline $\begin{array}{l}\text { MPAID (per } 1 \mathrm{~mm} \text { increased or } \\
\leq 21.5 \mathrm{~mm} \text { ) }\end{array}$ & 0.68 & $0.55-0.83$ & $<.001$ & 4.3 & $1.89-9.8$ & .001 \\
\hline $\operatorname{LVEDV}(\mathrm{mL})$ & 0.99 & $0.97-1.02$ & .55 & 0.98 & $0.96-1.01$ & .14 \\
\hline Previous No. of PVA & 3.52 & $0.48-25.82$ & .22 & 5.61 & $0.64-49.26$ & .12 \\
\hline $\begin{array}{l}\text { Previous functional length of } \\
\text { AVFs (mo) }\end{array}$ & 0.91 & $0.80-1.02$ & .12 & 0.89 & $0.78-1.01$ & .07 \\
\hline $\begin{array}{l}\text { Previous functional length of } \\
\text { tcCVCs (mo) }\end{array}$ & 0.81 & $0.62-1.07$ & .14 & 0.82 & $0.63-1.09$ & .17 \\
\hline $\begin{array}{l}\text { Previous duration of FV- } \\
\operatorname{ntCVC}(\mathrm{mo})\end{array}$ & 1.27 & $0.96-1.66$ & .09 & 1.19 & $0.91-1.56$ & .2 \\
\hline $\begin{array}{l}\text { Previous duration of IVJ- } \\
\mathrm{ntCVC}(\mathrm{mo})\end{array}$ & 1.02 & $0.78-1.34$ & .89 & 0.96 & $0.72-1.27$ & .75 \\
\hline Right RCAVF & 3.23 & $1.09-9.55$ & .034 & 2.95 & $1.02-8.54$ & .047 \\
\hline
\end{tabular}

TABLE 4 Cox proportional hazard regression models for RCAVF abandonment

Abbreviations: DBP, diastolic blood pressure; FV, femoral vein; $\mathrm{HR}$, Hazard rate; $\mathrm{Cl}$, confidence interval; IJV, internal jugular vein; LVEDV, left ventricle end-diastolic volume; LVID, left ventricle internal diameter; MPAID, main pulmonary artery internal diameter; ntCVC, nontunneled central venous catheter; PVA, permanent vascular access; RCAVF, radiocephalic arteriovenous fistula; tcCVC, tunneled cuffed central venous catheter. model a (MPA as continuous variable): Overall (score) $\chi^{2}=35.16, P=.001$. model $\mathrm{b}$ (added MPA $\leq 21.5 \mathrm{~mm}$ ): Overall (score) $\chi^{2}=37.13, p<.001$.

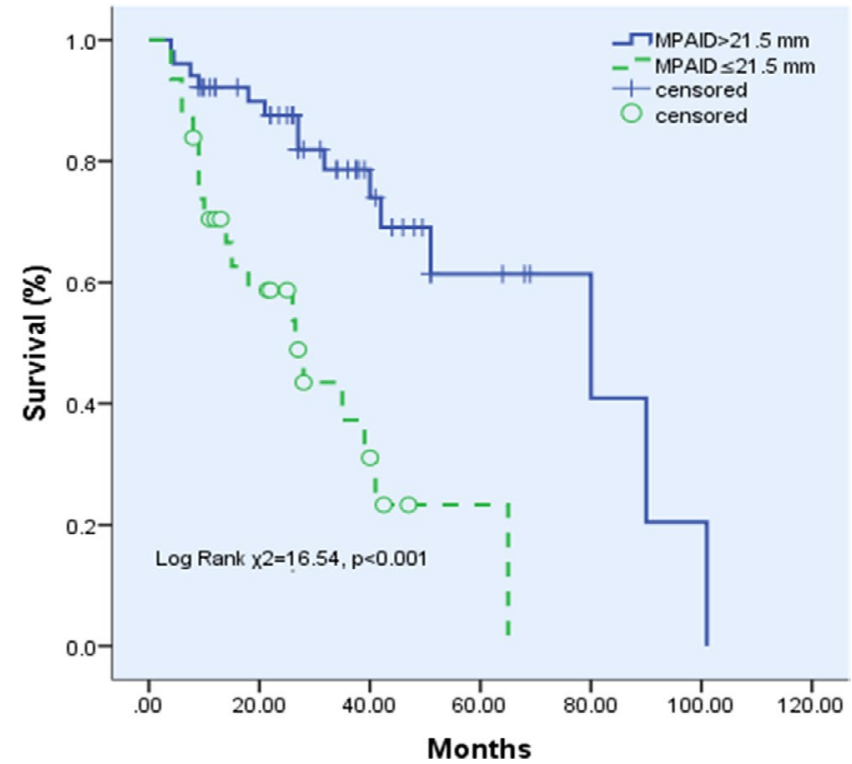

FIGURE 2 Curves respectively illustrating the cumulative survival of RCAVF (equal to cumulative patency) during study period in groups according to the value of MPAID. MPAID, main pulmonary artery internal diameter [Color figure can be viewed at wileyonlinelibrary.com]

our subjects had very few cardiovascular disorders (Table 1) and EF $\geq 50 \%$, as inferior AVF blood flow was observed in patients with abnormal EF. 22
The main reason for RCAVF dysfunction in the study was AVF stenosis (95\%). However, only 15 (35.7\%) patients received endovascular or surgical interventions. Most of the others missed the opportunity for early treatment, which led to an abandonment rate of $41.5 \%$ during the study period. Generally, delayed detection and referral made it difficult to rescue the dysfunctional AVFs.

\section{3 | Limitations}

The present study had some limitations. Firstly, there was a small sample size and some inherent bias in its retrospective nature, which means we could not rule out selective bias of patients or acquire the entirety of relevant events or precise information such as medication, dialysis prescription, and nutrition status during the study period. Although patients in this study visited our clinic regularly and underwent each operation in the same surgical team, they did not receive dialysis treatments in the same HD center. Additionally, we lacked echocardiographic data for the endpoint to further verify the association between the changes of cardiovascular system and the function of AVF. Secondly, the deviation from the baseline data might weaken the reliability of the study. The echocardiography was performed using the same machine in our hospital by skilled specialists, not the same technician. Lastly, the study population consisting of Chinese individuals did not reflect a multiethnic cohort. Hence, the results cannot be generalized to other ethnic groups or a general population. 


\section{5 | CONCLUSIONS}

In the retrospective study of 82 elderly Chinese patients with RCAVF on HD, LVEDV, and MPAID assessed by echocardiography showed significant predictive performance for the dysfunction and abandonment of RCAVF respectively, after adjustment for modality and duration of VA prior to RCAVF creation. The right side of RCAVF was also a risk factor both for dysfunction and abandonment. Significant differences existing between the Chinese population and others with respect to the patency of AVF necessitate additional studies.

\section{ACKNOWLEDGMENTS}

The data reported herein were supplied by West China Hospital of SiChuan University. The interpretation and reporting of these data are the responsibility of the authors and do not reflect the official views or opinions.

\section{CONFLICT OF INTEREST}

None.

\section{AUTHOR CONTRIBUTIONS}

Ping Fu and Yang Yu designed this study; Chunle Zhang, Liya Wang, and Yuchen Deng contributed to the acquisition of data; Yuqin Xiong analyzed and interpreted the data; Yang Yu and Yuqin Xiong drafted the manuscript; Emily Morris, Yi Li, and Ping Fu revised the manuscript for important intellectual content.

\section{STATEMENT OF ETHICS}

This study adheres to the Declaration of Helsinki and was approved by the ethics committee (No. 2017204). Written informed consent was obtained from each patient.

\section{ORCID}

Ping Fu iD https://orcid.org/0000-0002-3061-5925

\section{REFERENCES}

1. Saran R, Li YI, Robinson B, et al. US Renal Data System 2015 Annual Data Report: Epidemiology of Kidney Disease in the United States. Am J Kidney Dis. 2016;67(3 Suppl 1):Svii S1-305.

2. Arhuidese IJ, Cooper MA, Rizwan M, Nejim B, Malas MB. Vascular access for hemodialysis in the elderly. J Vasc Surg. 2019;69(2):517525.e1.

3. Arhuidese IJ, Orandi BJ, Nejim B, Malas M, Malas M. Utilization, patency, and complications associated with vascular access for hemodialysis in the United States. J Vasc Surg. 2018;68(4):1166-1174.

4. Reddy YNV, Obokata M, Dean PG, Melenovsky V, Nath KA, Borlaug BA. Long-term cardiovascular changes following creation of arteriovenous fistula in patients with end stage renal disease. Eur Heart J. 2017;38(24):1913-1923.

5. Basile C, Lomonte C. The complex relationship among arteriovenous access, heart, and circulation. Semin Dial. 2018;31(1):15-20.
6. Reque J, Garcia-Prieto A, Linares T, et al. Pulmonary hypertension is associated with mortality and cardiovascular events in chronic kidney disease patients. Am J Nephrol. 2017;45(2):107-114.

7. Wen M, Li Z, Li J, et al. Risk factors for primary arteriovenous fistula dysfunction in hemodialysis patients: a retrospective survival analysis in multiple medical centers. Blood Purif. 2019;48(3):276-282.

8. Schmidli J, Widmer MK, Basile C, et al. Vascular access: 2018 Clinical practice guidelines of the European society for vascular surgery (ESVS). Eur J Vasc Endovasc Surg. 2018;55(6):757-818.

9. Adequate hemodialysis work group of Chinese medical doctor association. Clinical practice guidelines for adequate hemodialysis in china. Natl Med J China. 2015;34:2748-2753.

10. Shenoy S, Allon M, Beathard G, et al. Clinical trial end points for hemodialysis vascular access: background, rationale, and definitions. Clin J Am Soc Nephrol. 2018;13(3):490-494.

11. Aitken E, Kerr D, Geddes C, Berry C, Kingsmore D. Cardiovascular changes occurring with occlusion of a mature arteriovenous fistula. $J$ Vasc Access. 2015;16(6):459-466

12. Yu Y, Xiong Y, Zhang C, Fu M, Li Y, Fu P. Vascular Access Type Was Not Associated with Mortality and the Predictors for Cardiovascular Death in Elderly Chinese Patients on Hemodialysis. Blood Purif. 2019;2:1-8. Epub ahead of print.

13. Basile C, Lomonte C, Vernaglione L, Casucci F, Antonelli M, Losurdo $\mathrm{N}$. The relationship between the flow of arteriovenous fistula and cardiac output in haemodialysis patients. Nephrol Dial Transplant. 2008;23(1):282-287.

14. Said K, Hassan M, Farouk M, Baligh E, Zayed B. Right ventricular function after creation of an atriovenous fistula in patients with end stage renal disease. Heart Lung Circ. 2019;28(6):884-892.

15. Zamboli $\mathrm{P}$, Lucà $\mathrm{S}$, Borrelli $\mathrm{S}$, et al. High-flow arteriovenous fistula and heart failure: could the indexation of blood flow rate and echocardiography have a role in the identification of patients at higher risk? J Nephrol. 2018;31(6):975-983.

16. Misskey J, Faulds J, Sidhu R, Baxter K, Gagnon J, Hsiang Y. An agebased comparison of fistula location, patency, and maturation for elderly renal failure patients. J Vasc Surg. 2018;67(5):1491-1500.

17. Wolowczyk L, Williams AJ, Donovan KL, Gibbons CP. The snuffbox arteriovenous fistula for vascular access. Eur J Vasc Endovasc Surg. 2000;19:70e6.

18. Hwang D, Park S, Kim HK, Huh S. Comparative outcomes of vascular access in patients older than 70 years with end-stage renal disease. J Vasc Surg. 2019;69(4):1196-1206.e5.

19. Goh MA, Ali JM, lype S, Pettigrew GJ. Outcomes of primary arteriovenous fistulas in patients older than 70 years. J Vasc Surg. 2016;63(5):1333-1340.

20. Brunori G, Ravani P, Mandolfo S, Imbasciati E, Malberti F, Cancarini G. Fistula maturation: doesn't time matter at all? Nephrol Dial Transplant. 2005;20(4):684-687.

21. Wakabayashi $\mathrm{K}$, lo $\mathrm{H}$, Nakata J, et al. Effects of cardiac function with postoperative arteriovenous fistula blood flow in patients with hemodialysis. Blood Purif. 2017;44(1):24-29.

22. Rayner HC, Pisoni RL, Gillespie BW, et al. cannulation and survival of arteriovenous fistulae: data from the Dialysis Outcomes and Practice Patterns Study. Kidney Int. 2003;63(1):323-330.

How to cite this article: Xiong Y, Yu Y, Zhang C, et al. The role of echocardiography in prognosis for dysfunction and abandonment of radiocephalic arteriovenous fistula in elderly Chinese patients on hemodialysis. Semin Dial. 2020;33:309315. https://doi.org/10.1111/sdi.12871 